\title{
Tendinose do supra-espinhoso em cães
}

\section{Supraspinatus tendinosis in dogs}

\author{
Maria Lígia de Arruda Mistieri ${ }^{1 *}$; Martin Kramer $^{2}$; Júlio Carlos Canola ${ }^{3}$; \\ João Guilheme Padilha Filho ${ }^{4}$
}

\section{Resumo}

A tendinose do supra-espinhoso é uma desordem recentemente relatada em cães e bem documentada em seres humanos, espécie na qual representa $51 \%$ das causas de alteração na articulação do ombro. Em cães, a afecção pode levar à dor na articulação acometida e claudicação ou permanecer assintomática. $\mathrm{O}$ significado clínico do problema ainda não foi definido na espécie canina e parece ser subestimado. $\mathrm{O}$ objetivo deste trabalho é discorrer sobre as possíveis etiologias desta tendinopatia e principais métodos diagnósticos, bem como abordar as terapias mais aplicadas atualmente em medicina veterinária, atentando ao clínico sobre as características da doença e a importância de incluí-la no diagnóstico diferencial de causas de dor e claudicação do membro anterior em cães.

Palavras-chave: Tendinose., tendão supra-espinhoso, cão

\begin{abstract}
Supraspinatus tendinosis was recently reported in dogs but it is a well-documented disorder in human beings, specie in which is considered the cause of pain and dysfunction in $51 \%$ of cases of shoulder problems. It can cause pain and lameness or be asymptomatic in dogs. The clinical relevance in canine specie is unclear and seems to be underestimated. The aim of this paper is to review the possible etiologies involved in this tendinopathy, the main diagnostic methods applied and actual options of therapies in veterinary medicine. Simultaneously, call the clinicians attention to this disorder as differential diagnosis for forelimb pain and lameness diseases.
\end{abstract}

Key words: Tendinosis, supraspinatus tendon, dog

1 Professora Colaboradora, UDESC, Campus de Lages. E-mail: malimistieri@gmail.com

2 Professor Titular Universidade de Giessen, Alemanha

3 Professor Livre Docente, UNESP, Campus de Jaboticabal

4 Professor Assistente Doutor, UNESP, Campus de Jaboticabal

* Autor para correspondência 


\section{Introdução}

A etiologia da dor no ombro é considerada um desafio diagnóstico em cães, pois ampla variedade de causas podem levar à dor e disfunção semelhantes, especialmente quando relacionadas a tecidos moles (ALASAARELA et al., 1997; BEALE, 2002).

Considerando-se as anormalidades de tecidos moles do ombro, a tendinose calcificante do supra-espinhoso foi descrita em cães no início da década de 90 como lesão relativamente rara e de relevância clínica obscura (KRIEGLEDER, 1995; PIERMATTEI; FLO, 1999). No entanto, em seres humanos a calcificação tendínea é bem conhecida, e sabidamente pode afetar diversos tendões; porém, é particularmente prevalente no tendão supraespinhoso e considerada a causa em $51 \%$ dos pacientes que apresentam dor ou disfunção do ombro (RE; KARZEL, 1993; MUIR; JOHNSON, 1994; MUIR et al., 1996; SPEED; HAZELMAN, 1999).

Em cães, a calcificação do tendão supraespinhoso pode resultar em dor e claudicação do membro torácico. No entanto, muitos animais permanecem clinicamente inalterados, apesar das evidências radiograficas de calcificação. Nestes casos, as calcificações são classificadas como achados radiográficos (FLO; MIDDLETON, 1990; SKVORAK, 1993; MUIR; JOHNSON, 1994).

O significado clínico e etiopatogenia da tendinose do supra-espinhoso não foram ainda determinados em cães, embora existam várias teorias sobre sua origem em seres humanos (FLO; MIDDLETON, 1990; KRIEGLEDER, 1995; SCHULZ, 2001). O diagnóstico da alteração em cães, não obstante, parece ser subestimado quando comparado à importância que representa na medicina. Objetivase, com a presente trabalho, revisar temas relevantes concernentes à afecção, incluido sua etiopatogenia, sintomatologia, diagnóstico e opções de tratamento disponíneis para aplicação em cães. Ressalta-se que não se encontra disponível, até o momento, uma revisão de literatura similar no meio científico brasileiro.

\section{Revisão}

A tendinose do supra-espinhoso foi primeiramente descrita em cães como de origem idiopática (MUIR et al., 1996). Ao longo dos anos, no entanto, teorias sobre sua etiologia foram descritas para espécie humana e, devido às semelhanças características do tendão supra-espinhoso e canino, tomadas como verdadeiras também para cães. Dentre tais hipóteses encontra-se a teoria vascular, sugerindo que tendões, por tratarem-se de tecidos metabolicamente ativos e requererem suprimento sanguíneo, poderiam desencadear degeneração secundária a um comprometimento vascular (REES; WILSON; WOLMAN, 2006). Reforçando esta teoria, estudos microangiográficos demonstraram apresentar o tendão supra-espinhoso, tanto de cães como de seres humanos, área hipovascular denominada "zona crítica", na qual encontramse, com maior prevalência, calcificações (RE; KARZEL, 1993; DANOVA; MUIR, 2003; REES; WILSON; WOLMAN, 2006). De acordo com a teoria vascular, hipóxia tendínea é considerada o fator inicial para deposição de materiais calcificados, como hidroxiapatita, cursando com degeneração fibrocartilaginosa (HAZELMAN, 1990; KJELLIN et al., 1991; ANDERSON; STEAD; COUGHLAN, 1993; RE; KARZEL, 1993; FARIN, 1996).

A teoria mecânica foi quase que simultaneamente proposta: preconizando que o sobreuso, excesso de peso e traumas repetitivos poderiam culminar em fadiga tendínea e, eventualmente, levarà degeneração de algumas áreas do tecido, posteriormente substituídas por calcificação distrófica (COFIELD, 1985; FLO; MIDDLETON, 1990; KJELLIN et al., 1991; PIERMATTEI; FLO, 1999). Segundo Muir e Johnson (1994), ambas as teorias acima descritas poderiam ocorrer conjuntamente, uma vez que lesões mecânicas podem alterar o suprimento vascular do tendão, acarretando hipóxia.

Em paralelo às teorias já discorridas, sabese que os tenócitos são células metabolicamente ativas que sintetizam e degradam continuamente 
a matriz tendína extracelular, em processo bem regulado e contínuo de metabolismo conhecido como "turnover". Um desbalanço deste delicado metabolismo pode também ser implicado no processo de degeneração tecidual (RILEY, 2005).

Além das causas acima descritas, predisposições genética, etária, sexual, laboral e até mesmo com relação à doenças sistêmicas concomitantes já foram reportadas em seres humanos, mas não ainda em cães (RILEY, 2005; REES; WILSON; WOLMAN, 2006).

$\mathrm{Na}$ realidade, o significado clínico da tendinose do supra-espinhoso em cães permanece tão obscuro quanto a sua fisiopatologia (MUIR; JOHNSON, 1994; KRIEGLEDER, 1995). Desde sua descrição, por serem muitos os casos assintomáticos, ainda não foi determinado nem ao menos quando a lesão causa ou não sinal clínico (MUIR et al., 1996; LAITINEN; FLO, 2000). Quando presentes, os sinais são relatados como claudicação leve a moderada, frequentemente observada em cães adultos de raças grandes, em especial rottweilers e labradores, com piora após exercícios ou ao longo do dia (ANDERSON; STEAD; COUGHLAN, 1993; MUIR; JOHNSON, 1994; KRIEGLEDER, 1995; MUIR et al., 1996; PIERMATTEI; FLO, 1999; LAITINEN; FLO, 2000). O exame clínico pode revelar ou não dor à manipulação do ombro afetado (KRIEGLEDER, 1995).

De acordo com Laitinen e Flo (2000), a presença de reação inflamatória ao redor da calcificação é possível causa de dor e claudicação em cães. Em seres humanos, a manifestação de sinais clínicos é descrita como dependente da fase da doença: a fase inicial, conhecida como de formação, é raramente sintomática; os sintomas estão relacionados a uma fase tardia, de reabsorção, quando há migração fagocitária e aumento de vascularização, resultando em edema, aumento de pressão intratendínea e, por consequência, dor (GARTNER; SIMONS, 1990; SEIL et al., 2006). A presença de fases da doença e sua possível relação com sinais clínicos não foi determinada em cães.
Kriegleder (1995) e Fransson, Gavin e Lahmers (2005) afirmaram que calcificações ou massas presentes no tendão supra-espinhoso, especialmente quando localizadas profundamente, podem ter contato com a bainha do tendão bíceps braquial e causar tenossinovite secundária, à qual por sua vez, levaria aos sinais clínicos.

Embora muitos cães sejam assintomáticos, a tendinose do supra-espinhoso já foi relatada como causa de dor e claudicação em 2,8 a 7\% dos cães com sinais clínicos em membro torácico (KRIEGLEDER, 1995; MUIR et al., 1996). Lafuente et al. (2007) referem que a claudicação é, geralmente, observada em apenas um dos membros, mas a alteração do tendão supra-espinhoso é comumente bilateral.

Para o diagnóstico de tendinose do supraespinhoso, há necessidade de realização de técnicas de imagem, iniciando-se com as radiografias. A avaliação radiográfica do ombro com tendinose do supra-espinhoso pode revelar presença de mineralização, representada por estruturas arredondadas, ovóides ou irregulares, radiopacas, próximas ao tubérculo maior e sulco intertubercular do úmero em projeções médio-laterais (Figura 1) (ANDERSON; STEAD; COUGHLAN, 1993; SKVORAK, 1993; KRIEGLEDER, 1995; PIERMATTEI; FLO, 1999; FARROW, 2003).

A projeção flexionada ou tangencial fornece detalhes do sulco intertubercular, otimizando a aparência da lesão e permitindo sua distinção quanto à localização no tendão do bíceps braquial, como descreveram Flo e Middleton (1990) e Piermattei e Flo (1999). O uso de meio de contraste (artrografia) permite diferenciar com maior segurança a localização da calcificação no tendão supraespinhoso, bíceps braquial ou sulco intertubercular (ANDERSON; STEAD; COUGHLAN, 1993). A realização de radiografias também é importante para a exclusão de outras possíveis causas de claudicação e dor mais comuns que a tendinose do supra-espinhoso; aconselha-se sua realização em ambos os membros (LAITINEN; FLO, 2000). 


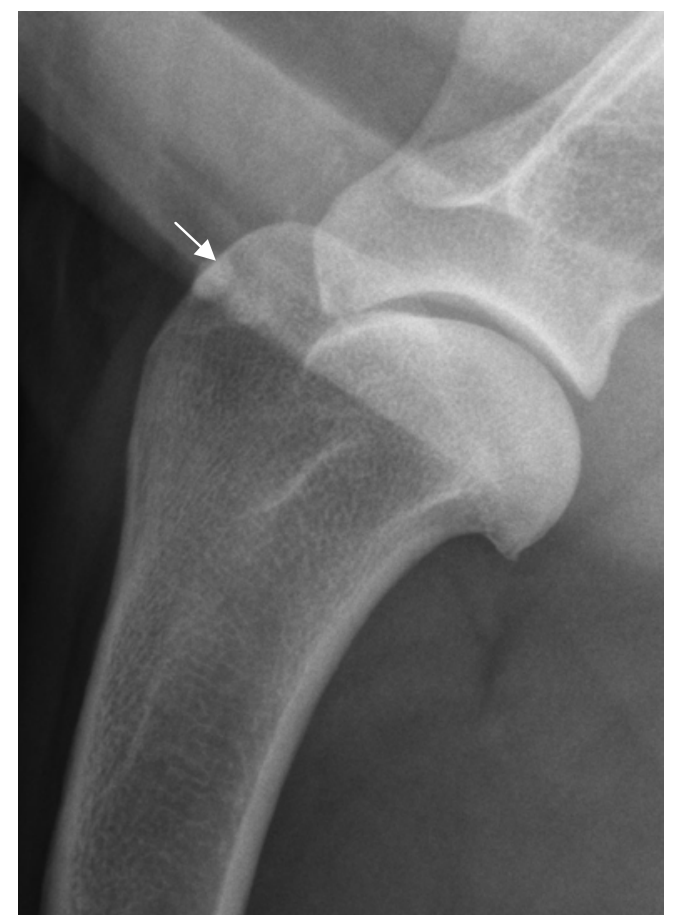

Figura 1. Imagem radiográfica em projeção médiolateral de ombro de cão com suspeita de tendinose do supra-espinhoso. Notar estrutura radiopaca (seta branca) de formato ovalado, localizada sobre o tubérculo maior do úmero.

Em se tratando de avaliação de tecidos moles, informações fornecidas por radiografias e artrografias são muito limitadas (SCHAEFER; FORREST, 2006). Nos últimos 20 anos, a ultrassonografia é crescentemente aplicada no diagnóstico de lesões em tecidos moles do ombro em seres humanos (ASARAAELA et al., 1997). Embora estudos prévios tenham indicado ser a ultrassonografia relativamente insensível (RIVERS; WALLACE; JOHNSTON, 1992), com o advento de transdutores de melhor resolução, a técnica provou proporcionar muitas informações em investigações de injúrias de tecidos moles do ombro. A ultrassonografia músculo-esquelética é amplamente utilizada em seres humanos, particularmente em avaliações do ombro, e tem mostrado crescente valor na rotina de pequenos animais (STILES; OTTE, 1993; TYSON, 1995; KRAMER et al., 1997, 2001; LONG; NYLAND, 1999; BRUCE et al., 2000; TEEFEY et al., 2000; IANNOTTI et al., 2005).

A precisão diagnóstica da ultrassonografia na avaliação de tecidos moles do ombro em seres humanos é comparável com a da ressonância magnética (TEEFEY et al., 2004), caracterizandose a primeira como método de escolha devido ao seu baixo custo de manutenção (KRAMER et al., 1999; BRUCE et al., 2000). A avaliação tendínea por ultrassonografia permite a diferenciação da lesão em ruptura parcial ou total, presença de tecido cicatricial, abscesso, metaplasia, neoplasia, inflamação e calcificação (GERWING; KRAMER, 1994; KRAMER et al., 1997, 2001; LAITINEN; FLO, 2000; (DINNES et al., 2003).

Como descrito por muitos autores, tendões e tecidos circujacentes são melhores avaliados com transdutores lineares de, no mínimo, 7,5 e 10 MHz com o animal sob anestesia geral ou sedação profunda, pois o exame inclui avaliação em posições de repouso e dinâmicas, por vezes desconfortáveis (KRAMER et al., 1997, 2001; LONG; NYLAND, 1999).

A aparência sonográfica da tendinose do supraespinhoso em cães foi descrita por Gerwing e Kramer (1994) como presença de calcificação nítida, acompanhada ou não de sombreamento acústico distal, geralmente sem reação circundante, localizada entre a transição músculo-tendínea (Figura 2). Os mesmos autores afirmam ser a avaliação ultrasonográfica fundamental para assegurar em qual tendão ou músculo se localiza a lesão e para avaliar sua extensão e aparência. 


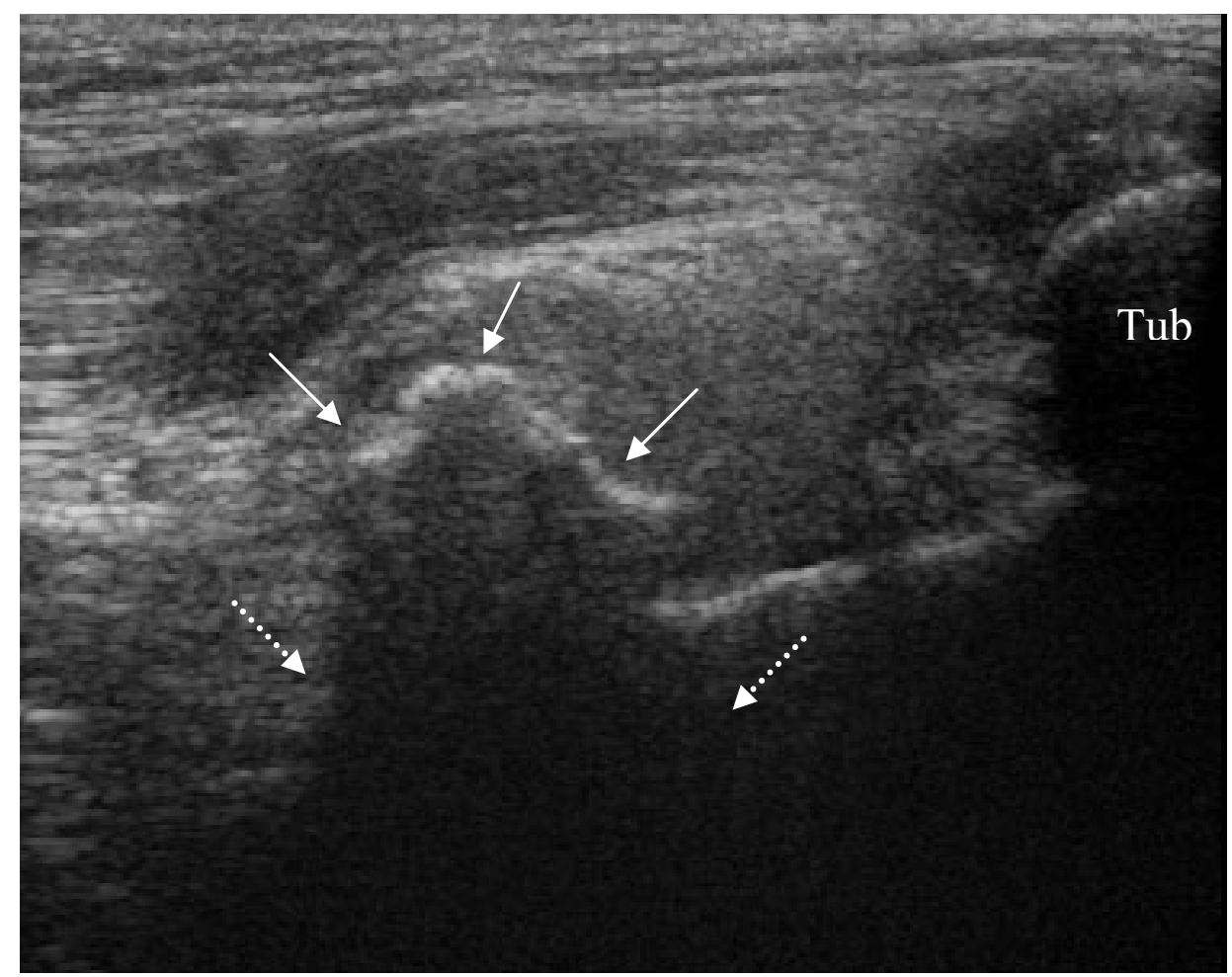

Figura 2. Imagem ultrassonográfica do tendão supra-espinhoso de cão com tendinose do supra-espinhoso, em plano longitudinal, obtida com trasdutor linear de $12 \mathrm{MHz}$. Notar estrutura irregular hiperecóica (setas brancas) acompanhada de sombreamento acústico distal (setas pontilhadas), localizado na transição músculo tendínea. O tubérculo maior, onde se insere o tendão, está identificado como "Tub" e representa a parte mais proximal da imagem.

Recentemente Fransson, Gavin e Lahmers (2005) relataram presença de massa no tendão supra-espinhoso de cão como causa de tenossinovite bicipital, diagnosticada e bem detalhada por meio de ressonância magnética. Embora a técnica seja amplamente empregada na medicina e considerada de escolha em avaliação de tendões e ligamentos, nenhum outro trabalho relacionando avaliação de tendinose do supra-espinhoso em cães por meio de ressonância magnética foi encontrado e sua aplicação ainda é limitada pelo alto custo (LONG; NYLAND, 1999; ZLATKIN, 2006).

$\mathrm{Na}$ prática, utiliza-se a radiografia simples em projeção médio lateral do ombro como exame de triagem. Quando da suspeita de tendinose do supraespinhoso, realiza-se a ultrassonografia tendínea que confirma sua presença no tendão examinado, bem como fornece informações adicionais tais como a estensão da lesão e se existe reação inflamatória circunjacente, caracterizada hipoecogenicidade do tecido ao redor da calcificação. Com as informações obtidas por meio dos dois exames, descartase a necessidade de procedimentos invasivos, como a artrografia, ou caros, como a ressonância magnética.

O tratamento de tendinose do supra-espinhoso em cães pode ser medicamentoso ou cirúrgico. A prescrição de anti-inflamatórios não esteroidais ou esteroidais por períodos longos (média de seis semanas) associada a repouso é bastante utilizada (MUIR; JOHNSON, 1994; KRIEGLEDER, 1995; PIERMATTEI; FLO, 1999). Administração local de acetato de metilprednisolona associada a repouso representou sucesso apenas temporário, como demonstrado por Kriegleder (1995).

Danova e Muir (2003) utilizaram terapia de choque extra-corpóreo em dois cães, como alternativa à remoção cirúrgica da calcificação, e 
observaram melhora clínica da claudicação. Em outro cão portador de tenossinovite bicipital, a técnica tambpem trouxe benefícios e melhora da claucicação (VENZIN et al., 2004). Este método está bastante difundido no tratamento de pacientes humanos desde 1992 e garante analgesia prolongada (HAAKE et al., 2002). Entretanto, nenhum estudo padronizado envolvendo este método foi realizado em cães até o presente momento.

Laitinen e Flo (2000) concluíram que o tratamento conservativo deve ser tentado por pelo menos três meses antes de se optar por excisão cirúrgica da calcificação do tendão supra-espinhoso em cães. Embora o tratamento cirúrgico seja considerado de fácil execução e consista da excisão do material calcificado por meio de pequena incisão longitudinal do tendão supra-espinhoso, pode não levar à melhora clínica em alguns casos, e por isso há receio em sua indicação. Lafuente et al. (2007) discorda, no entanto, da afirmação anterior por ter observado, em seu estudo retrospectivo, melhora clínica na maioria dos cães tratados cirurgicamente.

Laitinen e Flo (2000) observaram também elevado índice de recidivas de mineralizações nos tendões supra-espinhoso de cães operados, sem obrigatoriamente apresentarem sinais clínicos. Nenhum dos métodos de tratamento descritos é considerado ideal. Acredita-se que a afecção ainda é pouco estudada e a carência de estudos que incluam, principalmente, a avaliação dos casos tratados a longo prazo dificulta concluir qual método é o mais adequado (MUIR et al., 1996).

\section{Conclusão}

A tendinose do supra-espinhoso é uma afecção pouco conhecida em cães e que deve ser incluída no diagnóstico diferencial de causas de dor e claudicação do membro torácico. A divulgação de como a alteração é diagnosticada, bem como das possíveis opções de tratamento nesta espécie, é fundamental para que a alteração não continue a ser subestimada na rotina de pequenos animais.

\section{Agradecimentos}

À CAPES, pela bolsa de doutorado no Brasil e no exterior;

À FAPESP, pelo auxílio pesquisa;

À Universidade de Giessen, Alemanha, pelo aceite para realização do doutorado-sanduíche.

\section{Referências}

ALASAARELA, E.; TAKALO, R.; TERVONEN, O.; HAKALA, M.; SURAMO, I. Sonography and MRI in the evaluation of painful arthritic shoulder. British Journal of Rheumatology, Oxford, v. 36, n. 9, p. 996-1000, 1997.

ANDERSON, A.; STEAD, A. C.; COUGHLAN, A. R. Unusual muscle and tendon disorders of the forelimb in the dog. Journal of Small Animal Practice, Oxford, v. 34, n. 7, p. 313-318, 1993.

BEALE, B. Supraspinatus tendon injuries. In: THE WORLD ORTHOPAEDIC VETERINARY CONGRESS, 1., 2002, Munich. Proceedings... Munich: European Society of Veterinary Orthopaedics and Traumatology, Veterinary Orthopedic Society, 2002. p. 51.

BRUCE, W. J.; BURBIDGE, H. M.; BRAY, J. P.; BROOME, C. J. Bicipital tendinitis and tenosynovitis in the dog: a study of 15 cases. New Zealand Veterinary Journal, Wellington, v. 48, n. 2, p. 44-52, 2000.

COFIELD, R. H. Rotator cuff disease of the shoulder. Journal of Bone and Joint Surgery, Boston, v. 67, p. 974975, 1985.

DANOVA, N. A.; MUIR, P. Extracorporeal shock wave therapy for supraspinatus calcifying tendinopathy in two dogs. Veterinary Record, London, v. 152, n. 7, p. 208209, 2003.

DINNES, J.; LOVEMAN, E.; MCINTYRE, L.; WAUGH, $\mathrm{N}$. The effectiveness of diagnostic tests for the assessment of shoulder pain due to soft tissue disorders: a systematic review. Health Technology Assessment, Winchester, v. 7, n. 29, p. 1-166, 2003.

FARIN, P. U. Consistency of rotator -cuff calcifications: observations on plain radiography, sonography, computed tomography, and at needle treatment. Investigative Radiology, Hagerstown, v. 31, n. 5, p. 300-304, 1996.

FARROW, C. H. Veterinary diagnostic imaging: the dog and cat. Saint Louis: Mosby, 2003. v. 1. 
FLO, G. L.; MIDDLETON, D. Mineralization of the supraspinatus tendon in dogs. Journal of the American Veterinary Medical Association, Ithaca, v. 197, n. 1, p.9597, 1990.

FRANSSON, B. A.; GAVIN, P. R.; LAHMERS, K. K. Supraspinatus tendinosis associated with biceps brachii tendon displacement in a dog. Journal of the American Veterinary Medical Association, Ithaca, v. 227, n. 9, p. 1429-1433, 2005.

GARTNER, J.; SIMONS, B. Analysis of calcified deposits is calcifying tendinitis. Clinical Orthopaedics, Philadelphia, n. 254, p. 111-120, 1990.

GERWING, M.; KRAMER, M. Die Sonographie der Schlutergelenkes und seiner umgebenden Weichteile beim Hund. Kleintierpraxis, Hannover, v. 39, n. 3, p. 141-156, 1994.

HAAKE, M.; DEIKE, B., THON, A.; SCHMITT, J. Exact focusing of extracorporeal shock wave therapy for calcifying tendinopathy. Clinical Orthopaedics and Related Research, Philadelphia, n. 397, p. 323-331, 2002.

HAZELMAN, B. Why the supraspinatus tendon such a common site for calcification? British Journal of Rheumatology, London, v. 29, n. 5, p. 373, 1990.

IANNOTTI,J.P.;CICCONE,J.;BUSS,D.D.;VISOTSKY, J. L.; MASCHA, E.; COTMAN, K.; RAWOOL, N. M. Accuracy of office-based ultrasonography of the shoulder for the diagnosis of rotator cuff tears. Journal of Bone and Joint Surgery, Boston, v. 87a, n. 6, p. 1305-1311, 2005.

KJELLIN, M. D. I.; HO, C. P.; CERVILlA, V.; HAGHIGHI, P.; KERR, R.; VANGNESS, C. T.; FRIEDMAN, R. J.; TRUDELL, D.; RESNICK, D. Alterations in the supraspinatus tendon at MR imaging: correlation with histopathologic findings in cadavers. Radiology, Easton, v. 181, n. 3, p. 837-841, 1991.

KRAMER, M.; STENGEL, H.; GERWING, M.; SCHIMKE, E.; SHEPPARD, C. Sonography of the canine stifle. Veterinary Radiology \& Ultrasound, Raleigh, v. 40, n. 3, p. 282-293, 1999.

KRAMER, M.; GERWING, M.; HACH, V.; CHIMKE, E. Sonography of the musculoskeletal system in dogs and cats. Veterinary Radiology \& Ultrasound, Raleigh, v. 38, n. 2, p. 139-149, 1997.

KRAMER, M.; GERWING, M.; SHEPPARD, C.; SCHIMKE, E. Ultrasonography for the diagnosis of diseases of the tendon and tendon sheath of the biceps brachii muscle. Veterinary Surgery, Orlando, v. 30, n. 1, p. 64-71, 2001.
KRIEGLEDER, H. Mineralization of the supraspinatus tendon: clinical observations in seven dogs. Veterinary and Comparative Orthopaedics and Traumatology, Stuttgart, v. 8, n. 2, p. 91-97, 1995.

LAFUENTE, P.; FRANSSON B.; LINCOLN, J.; MARTINEZ, S.; LAHMERS, K. Surgical treatment of mineralized and non-mineralized supraspinatus tendinopathy in dogs: a retrospective study. In: ANNUAL SCIENTIFIC MEETING, 16., 2007, Dublin. Proceedings... Dublin: ECVS, 2007. p. 291-292.

LAITINEN, O. M.; FLO, G. L. Mineralization of the supraspinatus tendon in dogs: a long-term folow-up. Journal of the American Animal Hospital Association, Lakewood, v. 36, n. 3, p. 262-267, 2000.

LONG, C.; NYLAND, T. Ultrasonographic evaluation of the canine shoulder. Veterinary Radiology \& Ultrasound, Raleigh, v. 40, n. 4, p. 372-379, 1999.

MUIR, P.; JOHNSON, K. A.; COOLEY, A. J.; MANLEY, P. A. Force-plate analysis of gait before and after surgical excision of calcified lesions of the supraspinatus tendon in two dogs. Veterinary Record, London, v. 139, n. 6, p. 137-139, 1996.

MUIR, P.; JOHNSON, K. A. Supraspinatus and biceps brachii tendinopathy in dogs. Journal of Small Animal Practice, Oxford, v. 35, n. 5, p. 239-243, 1994.

PIERMATTEI, D. L.; FLO, G. A articulação escápuloumeral. In: . Manual de ortopedia e tratamento das fraturas dos pequenos animais. 3. ed. São Paulo: Manole, 1999. p. 215-244.

RE, L. P.; KARZEL, R. P. Management os rotator cuff calcifications. The Orthopedic Clinics of North America, Philadelphia, v. 24, n. 1, p. 125-132, 1993.

REES, J. D.; WILSON, A. M.; WOLMAN, R. L. Current concepts in the management of tendon disorders. Rheumatology, Oxford, v. 45, n. 5, p. 508-521, 2006.

RILEY, G. P. Gene expression and matrix turnover in overused and demaged tendons. Scandinavian Journal of Medicine \& Science in Sports, Copenhagen, v. 15, n. 4, p. 241-251, 2005.

RIVERS, B.; WALLACE, L.; JOHNSTON, G. R. Biceps tenosynovitis in the dog - radiographic and sonographic findings. Veterinary and Comparative Orthopaedics and Traumatology, Stuttgart, v. 5, n. 2, p. 51-57, 1992.

SCHAEFER, S. L.; FORREST, L. J. Magnetic resonance imaging of the canine shoulder: an anatomic study. Veterinary Surgery, Orlando, v. 35, n. 8, p.721-728, 2006. 
SCHULZ, K. S. Forelimb lameness in the adult patient. Veterinary Clinics of North America: Small Animal Practice, Philadelphia, v. 31, n. 1, p. 85-99, 2001.

SEIL, R.; LITZENBURGER, H.; KOHN, D.; RUPP, S. Arthroscopic treatment of chronically painful calcifying tendinitis of the supraspinatus tendon. Arthroscopy: the Journal of Arthroscopic \& Related Surgery, New York, v. 22, n. 5, p. 521-527, 2006.

SKVORAK, J. P. What is your diagnosis? Mineralization of the supraspinatus tendon. Journal of the American Veterinary Medical Association, Ithaca, v. 202, n. 2, p. 319-320, 1993.

SPEED, C. A.; HAZELMAN, B. Calcific tendonitis of the shoulder. New England Journal of Medicine, Boston, v. 340, n. 20, p. 1582-1584, 1999.

STILES, R. G.; OTTE, M. T. Imaging of the shoulder. Radiology, Easton, v. 188, n. 3, p. 603-613, 1993.

TEEFEY, S. A.; HASAN, S. A.; MIDDLETON, W. D.; PATEL, M.; WRIGHT, R. W.; YAMAGUCHI, K. Ultrasonography of the rotator cuff: a comparision of ultrasonographic and arthroscopic findings in one hundred consecutives cases. Journal of Bone and Joint Surgery, Boston, v. 82a, n. 4, p. 298-504, 2000.

TEEFEY, S. A.; RUBIN, D. A.; MIDDLETON, W. A.; HILDEBOLT, C. F.; LEIBOLD, R. A.; YAMAGUCHI, $\mathrm{K}$. Detection and quantification of rotator cuff tears. Comparision of ultrasonographic, magnetic resonance imaging, and arthroscopic findings in senventy-one consecutive cases, Journal of Bone and Joint Surgery. American volume, Boston, v. 86a, n.4, p. 708-716, 2004.

TYSON, L. L. Imaging of the painful shoulder. Current Problems in Diagnostic Radiology, Chicago, v. 24, n. 3, p. 110-40, 1995.

VENZIN, C.; OHLERTH, S.; KOCH, D.; SPRENG, D. Extracorporeal shockwave therapy in a dog with chronic bicipital tenosynovitis. Schweizer Archiv für Tierheilkunde, Bern, v. 146, n. 3, p. 136-41, 2004.

ZLATKIN, M. B. Shoulder. In: EDELMAN, R. E.; HESSELINK, J. R.; ZLATKIN, M. B.; CRUES, J. V. (Ed.). Clinical magnetic resonance imaging. $3^{\text {th }}$ ed. Philadelphia: Saunders, 2006. v. 3. p. 3204-3222. 